\title{
Exercise Training And Molecular Markers: New Perspectives In The Rehabilitation Of COPD
}

\author{
M Garofano ${ }^{1}$, MM Ascoli ${ }^{1}$, M Ciriello $^{1}$, M Aliberti ${ }^{1}$ and M Calabrese ${ }^{2 *}$ \\ ${ }^{1}$ Società Italiana Fisioterapia e Riabilitazione (S.I.Fi.R.), Italy \\ ${ }^{2}$ President Società Italiana Fisioterapia e Riabilitazione (S.I.Fi.R.), Italy
}

Received: 眥 May 23, 2018; Published: 跙May 29, 2018

*Corresponding author: Calabrese Mariaconsiglia, Società Italiana Fisioterapia e Riabilitazione (S.I.Fi.R.), Italy

\begin{abstract}
The present work is based on a careful analysis of the literature in order to find out what the right type of exercise can be recommended to the patient with COPD. Starting from the basic assumption that exercise is itself a biological therapy, we have looked to molecules that are critically altered in these patients so as to constitute potential molecular markers. We have also seen if, how, and how much the exercise is able to determine a modulation of the levels of these molecules in a functional sense to improve the clinical condition of the patients.
\end{abstract}

Abbreviations: COPD: Chronic Obstructive Pulmonary Disease; GOLD: Global Strategy for the Diagnosis; ADL: Activity of Daily Living; ROS: Reactive Oxygen Species

\section{Introduction}

Chronic Obstructive Pulmonary Disease (COPD), as defined in the recent revision of the Global Strategy for the Diagnosis (GOLD) guidelines, is a common, preventable and treatable disease characterized by persistent respiratory symptoms and airflow limitation, which is due to airway and alveolar abnormalities usually caused by significant exposure to harmful particles or gases. The chronic limitation to airflow characteristic of COPD is caused in part by changes in small airways (obstructive bronchiolitis) and in part by parenchymal destruction (emphysema). In addition to the clinical evaluation of the symptomatic picture and a careful history, for the diagnosis of COPD it is necessary to perform a spirometry. The presence of a post-bronchodilator FEV1/ FVC ratio $<0.70$ confirms the presence of persistent bronchial obstruction [1]. Dyspnea is certainly an element that we find in all patients with COPD to varying degrees, and this triggers a vicious circle that leads patients to reduce physical activity, causing an even more precocious appearance of dyspnea and muscle fatigue with reduced performance in Activity of Daily Living (ADL). The classic rehabilitation proposal includes an intervention focused on exercise training, although there is still a lot to do to clearly define the right amount of exercise that is safe and effective for each patient [2]. We now know with certainty that physical exercise can be considered a biological therapy, and compared to drugs, it induces even more significant changes, modulating the expression of genes and proteins at multiple levels, thus triggering functional changes. Starting from this premise, the need arises to understand the mechanisms of action, timing, dosage and adverse effects. Taking a cue from the omich sciences, the omich-rehabilitation is therefore born, that is the discipline that applies to the rehabilitation the study of molecular mechanisms at the base of the recovery processes, plasticity and adaptation, in order to allow the rehabilitator to build a customized therapy program for the patient and at the same obtaining positive feedback from the markers to demonstrate the efficacy of the treatment in a clear manner [3]. From the analysis of the literature in order to find molecules that are critically altered in the patient with COPD and the possibility to be modified through therapeutic exercise, a central role is played by Sirtuins and Interleukin 6 (IL-6). Sirtuins are enzymatic proteins acting as histone deacetylases or mono- ribosyltransferases, involved in the modulation of phenomena such as aging, regulation of transcription, apoptosis, resistance to stress [4,5]. A lot of studies show the relationship between Sirtuins (mostly SIRT1) and the development of COPD and, at the same time, cell aging, apoptosis, 
chronic inflammation, recognize a common trigger represented by smoking cigarette, which results in an epigenetic alteration of the DNA intervening in the mechanism of histone acetylation / deacetylation, and methylation / demethylation involving Histone deacetylase 2/ SIRT1 (HDAC2 / SIRT1) [6]. Sirtuin levels are typically decreased in patients with COPD, in relation to epigenetic changes induced by cigarette smoking; mainly the $120 \mathrm{kDa}$ fragment decreases, playing role as potential molecular marker [7] Rajendrasozhan S [8] first showed that the level of Sirt1 decreases in the lungs and macrophages of patients with COPD and in smokers compared to non-smokers.

The reduction of Sirt1 leads to an increase in Nuclear Factor Kappa light chain enhancer of activated B cells (NF-kB) activity responsible for the greater release of pro-inflammatory molecules such as IL-6 and IL-8 (Interleukin 8) [9]. Cigarette smoke also induces a reduction in the levels of Sirt4, a mitochondrial sirtuinin, leading to an increase in NF-kB activity and in transcription of the molecules that allow the adhesion of leukocytes to the endothelium, amplifying, in this way, the inflammatory phenomenon and downstream oxidative stress [10]. A confirmation of the beneficial effects of Sirtuin in COPD is given by a study in which is administered a Sirtuin activator, resveratrol, which allows, to smooth muscle cells in the airways, to reduce the secretion of proinflammatory cytokines, as IL6 and IL8. This effect is even greater than that induced by corticosteroids (usually used in the pharmacological treatment of COPD) [11]. Although the relationship between Sirtuin, oxidative stress and exercise has been proven, no study has been performed to investigate this relationship in the COPD patient. The positive effects determined by the exercise therefore involve Adenosine monophosphate activated protein kinase (AMPK) in a manner dependent on the intensity of the exercise itself, and numerous are the signal transduction pathways passing through the activation of AMPK and SIRT1 involved in the regulation of the antioxidant system in order to protect from the Reactive Oxygen Species (ROS) depending damage [12]. On the other hand,IL-6 represents both a pro-inflammatory and anti-inflammatory cytokine secreted by $\mathrm{T}$ lymphocytes and macrophages able to stimulate the immune response, during infection or following an injury such as a burn or other tissue damage leading to inflammation [13]. IL-6 with antiinflammatory function is called "myochin", a cytokine produced by muscle whose concentration increases in response to muscle contraction. In a systemic review of 2015 [14] were analyzed several studies that included the IL6 assay in patients with COPD, and it was found that serum IL- 6 concentration is higher in subjects with COPD than in healthy controls, correlating with the reduction in lung function; but not all studies agree with this result. The antiinflammatory action of IL6, produced by muscle, causes an increase or in blood levels of anti- inflammatory cytokines, or in producing inhibitors for pro-inflammatory cytokine receptors, such as Interleukin 1 receptor antagonist (IL-1ra), IL-10 (Interleukin 10), and soluble Tumor Necrosis Factor Receptors (sTNF-R) [15].
The nitric oxide plays a central role in the regulation of such muscular secretion [15]. The level of muscular IL6 increases already after the first session of exercise, and then falls into chronic activity, this seems related to the possibility that the exercise itself can determine an increase in sensitivity to IL6. Sirtuin is the ring binding physical exercise and secretion of IL6 both antiinflammatory and pro-inflammatory. It has also been shown that resveratrol induces Sirt1 increase, because it is a positive modulator of Sirt1 as well as exercise. Now, it is possible to deduce that exercise in patients with COPD also leads to a reduction in inflammation by decreasing pro-inflammatory IL6. The reduction of inflammation is also linked to anti-inflammatory IL6 which is secreted in response to production of nitric oxide (16) which in turn is closely related to Sirtuin. In conclusion, the question is which exercise do you recommend to patients with COPD? From the literature we have established that the anti-inflammatory IL6 increases after a single session of exercise, but there is an equally rapid decrease, which does not allow an effective anti-inflammatory action, and the release of muscle IL6 is proportional to the intensity of exercise and to the number of fibers recruited. This could be the reason of the effectiveness of resistance training in the rehabilitation of COPD. Regarding Sirtuin increase, it is now certainly linked to the therapeutic physical exercise carried out in chronic to moderate intensity. The chronic aerobic exercise determines the reduction of inflammation and oxidative stress by modulating Sirt1 target antioxidant enzymes (Superoxide Dismutase and Catalase). The decrease in the inflammatory phenomenon is certainly central for the improvement of the clinical and symptomatic picture in the patient with COPD. There is a growing need to produce new, rigorous and well-conducted studies to investigate the therapeutic dose of exercise to be recommended to our patients with COPD based on the measurements of specific biomarkers.

\section{References}

1. (2017) Global Strategy for the Diagnosis, Management and Prevention of COPD, Global Initiative for Chronic Obstructive Lung Disease (GOLD).

2. McCarthy B, Casey D, Devane D, Murphy K, Murphy E, et al. (2015) Pulmonary rehabilitation for chronic obstructive pulmonary disease. Cochrane Database Syst Rev 23(2): CD003793.

3. Wagner AK (2011) Rehabilomics: a conceptual framework to drive biologics research. PM R 3(6 Suppl 1): S28-30.

4. North BJ, Verdin E (2004) Sirtuins: Sir2-related NAD-dependent protein deacetylases. Genome Biol 5(5): 224.

5. Yamamoto H, Schoonjans K, Auwerx J (2007) Sirtuin functions in health and disease. Mol. Endocrinol 21(8): 1745-1755.

6. Sundar IK, Yao H, Rahman I (2013) Oxidative stress and chromatin remodeling in chronic obstructive pulmonary disease and smokingrelated diseases Antioxid Redox Signal 18(15): 1956-1971.

7. Yanagisawa S, Papaioannou AI, Papaporfyriou A, Baker JR, Vuppusetty C, et al. (2017) Decreased Serum Sirtuin-1 in COPD. Chest 152(2): 343352.

8. Rajendrasozhan S, Yang SR, Kinnula VL, Rahman I (2008) SIRT1, an antiinflammatory and antiaging protein, is decreased in lungs of patients 
with chronic obstructive pulmonary disease. Am J Respir Crit Care Med 177(8): 861-870

9. Hwang JW, Rajendrasozhan S, Yao H, Chung S, Sundar IK, et al. (2011) FOXO3 deficiency leads to increased susceptibility to cigarette smokeinduced inflammation, airspace enlargement, and chronic obstructive pulmonary disease. Journal of Immunology 187(2): 987998.

10. Yonfeng Chen, Wang H, Luo G, Dai X, et al. (2014) SIRT4 inhibits cigarette smoke extracts-induced mononuclear cell adhesion to human pulmonary microvascular endothelial cells via regulating NF- $\mathrm{KB}$ activity. Toxicology letters 26(3): 320-327.

11. Knobloch J, Wahl C, Feldmann M, Jungck D, Strauch J, et al. (2014) Resveratrol attenuates the release of inflammatory cytokines from human bronchial smooth muscle cells exposed to lipoteichoic acid in chronic obstructive pulmonary disease. Basic Clin Pharmacol Toxicol 114(2): 202-209.

12. Liao ZY, Chen JL, Xiao MH, Sun Y, Zhao YX, et al. (2017) The effect of exercise, resveratrol or their combination on Sarcopenia in aged rats via regulation of AMPK/Sirt1 pathway. Exp Gerontol 98:177-183.

13. Wolf, S Rose-John, C Garbers (2014) Interleukin-6 and its receptors: a highly regulated and dynamic system. Cytokine. 70(1): 11-20.

14. Jia Wei, Xiong XF, Lin YH, Zheng BX, Cheng DY, et al. (2015) Association between serum interleukin- 6 concentrations and chronic obstructive pulmonary disease: a systematic review and meta-analysis. Peer J 3: e1199.

15. Bente K Pedersen, Mark A Febbraio (2008) Muscle as an Endocrine Organ: Focus on Muscle-Derived Interleukin-6. Physiological Reviews 88(4): 1379-1406.

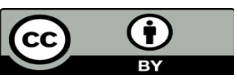

This work is licensed under Creative Commons Attribution 4.0 License

Submission Link: Submit Article

DOI: $10.32474 /$ DDIPIJ.2018.01.000119

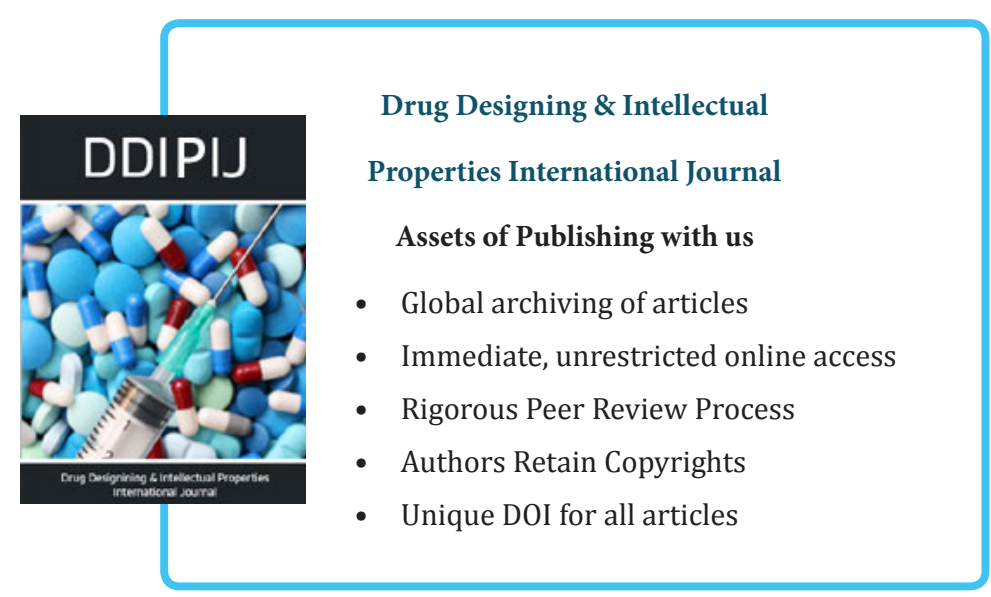

